\title{
Allicin induces apoptosis in gastric cancer cells through activation of both extrinsic and intrinsic pathways
}

\author{
WENLU ZHANG ${ }^{1,2}$, MINWEN HA ${ }^{2}$, YUEHUA GONG ${ }^{1}$, YING XU $^{1}$, NANNAN DONG $^{1}$ and YUAN YUAN ${ }^{1}$ \\ ${ }^{1}$ Cancer Control Laboratory of Cancer Institute and General Surgery, The First Affiliated Hospital of China Medical University, \\ Shenyang 110001; ${ }^{2}$ Department of Medical Oncology, The First Hospital, Liaoning Medical University, \\ Jinzhou, Liaoning 121001, P.R. China
}

Received May 12, 2010; Accepted July 20, 2010

DOI: $10.3892 /$ or_00001021

\begin{abstract}
Allicin is an active compound derived from garlic that has been shown to have antitumor properties in vitro. The current study was designed to explore the effects and the underlying mechanism of allicin on gastric cancer cells. The MTT assay was used to detect cell viability. Transmission electron microscopy, Rh123 and propidium iodide staining, Annexin V/FITC assay and the mitochondrial membrane potential were used to assess for the presence of apoptosis. Immunocytochemistry, Western blot analysis, and Q-RT-PCR were used to detect gene expression. We found that allicin reduced cell viability in a dose- and time-dependent manner, partly through induction of apoptosis in gastric cancer cells. At the molecular level, allicin induced cytochrome c release from the mitochondria and increased caspase- $3,-8$, and -9 activation, with concomitant upregulation of bax and fas expression in the tumor cells. Allicin treatment inhibited proliferation and induced apoptosis in SGC-7901 cancer cells. Both intrinsic mitochondrial and extrinsic Fas/FasL-mediated pathways of apoptosis occur simultaneously in SGC-7901 cells following allicin treatment. Data from the current study demonstrated that allicin should be further investigated as a novel cancer preventive or therapeutic agent in control of gastric cancer, with potential uses in other tumor types.
\end{abstract}

\section{Introduction}

Gastric cancer is the second leading cause of cancer-related deaths in the world, with an estimated $\sim 760,000$ cases of gastric cancer are diagnosed annually worldwide $(1,2)$. Gastric cancer is often asymptomatic, with only non-specific symptoms in its early stages. Thus, by the time symptoms

Correspondence to: Dr Yuan Yuan, Cancer Control Laboratory of Cancer Institute and General Surgery, the First Affiliated Hospital of China Medical University, Nanjing North Street \#155, Heping District, Shenyang 110001, P.R. China

E-mail: yyuan3@hotmail.com

Key words: Allicin, gastric cancer cells, apoptosis, extrinsic and intrinsic apoptosis pathways occur, the cancer has generally metastasized to other regions of the body. The cause of gastric cancer remains unknown, however, it is more common in 55 years or older men. In addition, a high salt diet and low vitamin intake increases the risk of gastric cancer. Pre-existing conditions, such as pernicious anemia, atrophic gastritis, and intestinal polyps also increase the risks and additional risk factors include hereditary non-polyposis colon cancer syndrome, Li-Fraumeni syndrome, a family history of gastrointestinal cancer, and blood type A. A diet high in raw fruits and vegetables, citrus fruits, and fiber may lower the risk for gastric cancer.

Surgery is still the first line of treatment for gastric cancer, with chemotherapy showing limited effectiveness. The prognosis of gastric cancer remains poor; nevertheless, if detected early, long-term survival of gastric cancer is very favorable $(1,2)$. Therefore, novel approaches for early detection, prevention, and treatment strategy are needed. Our research objective was to develop novel agents for the prevention and treatment of this deadly disease.

Garlic is widely used for its pungent flavor as a seasoning and in China, garlic has been consumed and used to treat various ailments for over three thousand years. More recently, epidemiological studies have shown that dietary intake of allium-containing food, such as garlic, decreased the risk of developing various types of malignancies (3-7), although the underlying molecular mechanisms remain to be defined. Allicin, diallyl thiosulfinate, is the main biologically active compound derived from garlic. It was first isolated and studied in the laboratory by C.J. Cavallito in 1944 where it was found to exhibit antibacterial and anti-fungal properties (8). It is formed by the enzyme alliinase on alliin (S-allyl-Lcysteine sulfoxide), alliin is chiral and only occurs as a racemic enzyme. This racemic form can also be generated by the oxidation of diallyl disulfide (9). Alliinase and alliin are enclosed in different compartments within the garlic cloves, with the intact garlic cloves not containing allicin. When the garlic clove is crushed, alliin and alliinase interact to form allicin, pyruvic acid and ammonia.

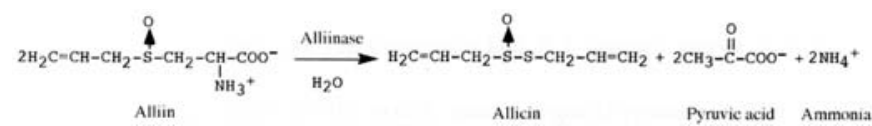

In a previous study by our group, allicin was demonstrated to induce mitotic arrest of gastric cancer cells (10). 
In the current study, we further investigated the effects of allicin on gastric cancer cells and evaluated its antitumor mechanism. We detected that allicin reduces tumor cell viability and apoptosis induction.

\section{Materials and methods}

Materials. Cell culture medium RPMI-1640, penicillin/ streptomycin, and trypsin were obtained from Gibco-BRL (Gaithersburg, MD). Fetal bovine serum (FBS) was from Solarbio Science \& Technology Co. Ltd. (Beijing, China). 3-(4,5-dimethyl thiazol-2-yl)-2,5-diphenyltetrazolium bromide (MTT) and dimethyl sulfoxide (DMSO) were purchased from Sigma-Aldrich (St. Louis, MO, USA). Annexin V-FITC and propidium iodide (PI) double staining kit and fluorescent dye rhodamine 123 (Rh123) were obtained from Key Gene (Nanjing, China). Anti-Bax, Fas, caspase-3, and cytochrome c antibodies and fluorescence-conjugated secondary antibodies were from Santa Cruz Biotechnology (Santa Cruz, CA, USA). Secondary antibodies and SP and DAB kits were from the Zhongshan Golden Bridge Biotechnology Co. Ltd., (Beijing, China). Real-time polymerase chain reaction (RTPCR) kits and gene-specific primers were from Takara (Bio Inc., Ohtsu, Japan). Caspase-3, -8, and -9 activity assay kits were from the Beyotime Institute of Biotechnology (Jiangsu, China). Allicin (HPLC purified to 98\%) was from Shandong Lukang Cisen Pharmtech Co. Ltd., (Shandong, China) and cisplatin (DDP) was from QILU Pharmaceutical Co. Ltd. (Jinan, Shandong, China).

Cell line and culture. The human gastric adenocarcinoma cell line SGC7901 was obtained from the Military Academy of Medical Science (Beijing, China). The cells were cultured in RPMI-1640 medium supplemented with $10 \%$ fetal bovine serum and $1 \%$ penicillin-streptomycin at $37^{\circ} \mathrm{C}$ in a humidified incubator containing $95 \%$ air and $5 \% \mathrm{CO}_{2}$. When cells reached $60-80 \%$ confluence, they were trypsinized with $0.25 \%$ trypsin, counted and seeded for drug treatments at the required density.

MTT assay. The effects of allicin on gastric cancer cell viability were assessed with the MTT assay. The cells were seeded at $1 \times 10^{4}$ per well in 96-well plates and grown overnight; they were then treated with different concentrations of allicin $(15-120 \mu \mathrm{g} / \mathrm{ml})$ for up to $72 \mathrm{~h}$. Thereafter, $20 \mu \mathrm{l}$ of MTT solution $(5 \mathrm{mg} / \mathrm{ml})$ was added to each well and the cells were incubated for an additional $4 \mathrm{~h}$ at $37^{\circ} \mathrm{C}$. The culture medium was then removed and the cells were lysed with $150 \mu \mathrm{l}$ of DMSO and the plate was shaken on a microvibrator for $10 \mathrm{~min}$ at room temperature. Optical density (OD) was then measured using a microplate reader (Bio-Rad, Hercules, CA, USA) at $570 \mathrm{~nm}$. The cell inhibition rate was calculated using the following formula: cell inhibition rate $(\%)=(1-\mathrm{OD}$ of treated culture/OD of the control culture $) \mathrm{x}$ $100 \%$.

Transmission electron microscopy (TEM). The tumor cells were grown and treated with different doses of allicin for $48 \mathrm{~h}$ and were then trypsinized. A total of $1 \times 10^{7}$ cells were pelleted by centrifugation at $2000 \mathrm{x}$ g for $5 \mathrm{~min}$ and washed twice with PBS by repeated centrifuging. The cells were then fixed in $2.5 \%$ ice-cold glutaraldehyde in $0.1 \mathrm{M}$ of sodium cacodylate/1\% sucrose buffer for $24 \mathrm{~h}$. Next, the cells were washed in triplicate with PBS and postfixed in $1 \%$ osmium tetroxide for $60 \mathrm{~min}$, encapsulated in $1 \%$ agar, and stained with uranyl acetate and phosphotungstic acid. The cells were then dehydrated in a series of graded ethanol incubated in propylene oxide and embedded in Epon 812-Araldite mixture. Ultrathin sections $(50 \mathrm{~nm})$ were cut using an LKL208 ultramicrotome and placed under 200 mesh standard copper grids, followed by HA-600 transmission electron microscopy analysis.

Rh123 and propidium iodide staining. The cells were grown and treated with $30 \mu \mathrm{g} / \mathrm{ml}$ of allicin for $48 \mathrm{~h}$ and then subjected to rhodamine 123 staining $(1 \mu \mathrm{g} / \mathrm{ml})$ at $37^{\circ} \mathrm{C}$ for $30 \mathrm{~min}$ and then with PI $(5 \mu \mathrm{l})$ for $5 \mathrm{~min}$. The cells were reviewed and scored under a fluorescence microscope.

Annexin V/FITC labeling. Allicin-induced gastric cancer cells undergoing apoptosis were further determined using an Annexin V/FITC apoptosis kit. Briefly, after treatment with $30 \mu \mathrm{g} / \mathrm{ml}$ of allicin for $48 \mathrm{~h}$, the cells were harvested by centrifuging at $1,000 \mathrm{rpm} / \mathrm{min}$, washed in duplicate with ice-cold PBS, pelleted, and resuspended at $1 \times 10^{6}$ concentration in $100 \mu \mathrm{l}$ of binding buffer $5 \mu \mathrm{l}$ of Annexin V-FITC conjugate and 10- $\mu 1$ PI solution were added to the cells and the cell solution was incubated for $15 \mathrm{~min}$ at room temperature in the dark followed by adding $300 \mu 1$ of the binding buffer. At least 10,000 cells were subjected to flow cytometer (Becton-Dickinson, Franklin Lakes, NJ, USA) analysis of the viable, apoptotic, and necrotic populations and quantified using CellQuest software according to the manufacturer's instructions.

Analysis of alterations in mitochondrial membrane potential following allicin treatment. The fluorescent dye rhodamine 123 was used to assess for changes in mitochondrial membrane potential following allicin treatment. Briefly, $1 \times 10^{6}$ cells were incubated with $30 \mu \mathrm{g} / \mathrm{ml}$ allicin for $48 \mathrm{~h}$. The cells were then resuspended, washed in duplicate with $\mathrm{PBS}$, and incubated with $5 \mu \mathrm{M}$ rhodamine 123 at $37^{\circ} \mathrm{C}$ for $30 \mathrm{~min}$. The cells were then analyzed using a FACStar flow cytometer and alterations in mitochondrial membrane potential were quantified using the ModFit software as described previously (11).

Immunocytochemistry. Expression of apoptosis-related proteins was evaluated using the immunocytochemical SP (avidin-chain-enzymatic oxidation) method. In brief, $5 \times 10^{4}$ SGC-7901 cells were cultured on glass coverslips overnight, and treated with $30 \mu \mathrm{g} / \mathrm{ml}$ of allicin for $48 \mathrm{~h}$. The cells were washed in duplicate with PBS and fixed with $4 \%$ paraformaldehyde in PBS for 15 min followed by permeabilization with $0.5 \%$ Triton X-100 in PBS for 3 min. After blocking with peroxide-methanol at room temperature, the cells were incubated with mouse anti-bax, cytochrome c, or caspase- 3 antibody at $4^{\circ} \mathrm{C}$ overnight. The following day, the cells were washed in triplicate with PBS and incubated with biotinylated rabbit anti-mouse IgG secondary antibody for $30 \mathrm{~min}$. A positive staining signal was developed by incubation of the 
cells in 3,3-diaminobenzidine (DAB) solution for $10 \mathrm{~min}$ at room temperature in the dark. The cells were then rinsed with distilled water and mounted. The coverslips were reviewed under a microscope and images were captured with an Olympus Q-imaging Micropublisher camera for quantification.

Protein extraction and Western blot analysis. The cells were grown and treated with allicin $(30 \mu \mathrm{g} / \mathrm{ml})$ for $48 \mathrm{~h}$ and then washed in PBS and suspended in a lysis buffer $(20 \mathrm{mM}$ HEPES, pH 7.9, 20\% glycerol, $200 \mathrm{mM} \mathrm{KCl,} 0.5 \mathrm{mM}$ EDTA, $0.5 \%$ NP40, $0.5 \mathrm{mM}$ DTT and $1 \%$ protease inhibitor cocktail). The lysates were collected and stored at $-20^{\circ} \mathrm{C}$ until further use. Supernatant protein concentration were determined using the Bradford method. The samples containing $40 \mu \mathrm{g}$ of total cellular protein were resolved with $6-10 \%$ of sodium dodecyl sulfate polyacrylamide gel electrophoresis depending on the size of the target proteins, transferred onto PVDF membranes by electroblotting, and probed with anticytochrome c, anti-Bax, anti-caspase-3, or anti-fas antibodies. After washing in PBS-T, the membranes were incubated with horseradish peroxidase-conjugated secondary antibodies. The membranes were then incubated in an enhanced chemiluminescencence solution and positive protein bands were visualized on X-ray film.

Quantification of caspase-3,-8 and -9 activity. Caspase activity was measured using caspase activity kits according to the manufacturer's instructions. Briefly, after treatment with allicin, the cells were washed with ice-cold PBS, resuspended in lysis buffer and incubated on ice for $15 \mathrm{~min}$. The lysate was centrifuged at $12,000 \mathrm{rpm} / \mathrm{min}$ for $15 \mathrm{~min}$ at $4^{\circ} \mathrm{C}$ and the activity of caspase- $3,-8$, and -9 was measured using the substrate peptides Ac-DEVD-p-nitroanilide (pNA), Ac-IETD-pNA and Ac-LEHD-pNA, respectively. The release of pNA was quantified by determining absorbance with the Multiskan Spectrum spectrophotometer (Thermo Scientific) at $405 \mathrm{~nm}$.

Quantitative real-time reverse transcriptase polymerase chain reaction ( $Q-R T-P C R)$. Expression of bax, cytochrome c, caspase-3, and fas was assessed using Q-RT-PCR. Briefly, after treatment with allicin, the cells were washed with icecold PBS and total RNA from the cells was isolated using TRIzol (Takara, Japan) and treated with DNase I (Takara). RNA was then reverse transcribed into cDNA for PCR amplification. The primers used were designed using the software Primer Premier 5.0 (Premier Co., CA) and synthesized by SBS Genetech Co., Ltd. The primers for Bax were: forward 5'-ATG GGC TGG ACA CTG GAC TTC-3', and reverse 5'-GAG CGA GGC GGT GAG GAC-3'. The primers for caspase-3 were: forward, 5'-GGAACGAACGG ACCTGTG-3' and reverse, 5'-GCCTCCACTGGTATCTT CTG-3'. The primers for Fas were: forward, 5'-TGATGTG GAACACAGCAAGG-3', and reverse, 5'-GGCTGTGGTG ACTCTTAGTGATAA-3'. The primers for cytochrome c were: forward, 5'-TCCTGGCAAAAGGTCAGAGT-3', and reverse, 5'-GTTGTGTGTTCGCCTCTTGA-3'. Total RNA was extracted by grinding the cells in TRIzol reagent (Takara) and any potential DNA contamination was removed by

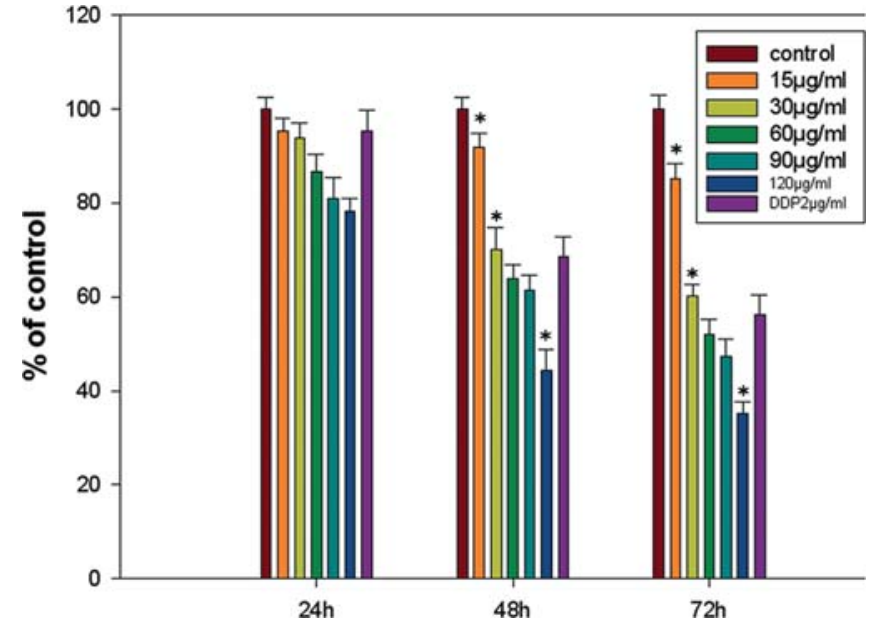

Figure 1. The effects of allicin in reducing gastric cancer cell viability. The cells were grown and treated with various concentrations of allicin for up to $72 \mathrm{~h}$. Cell viability was analyzed using the MTT assay. The data are presented as mean $\pm S E(n=6)$ of two independent experiments in triplicate. Cisplatin (DDP, $2 \mu \mathrm{g} / \mathrm{ml}$ ) was used as a positive control. ${ }^{*} \mathrm{P}<0.05$ between the control and treated cells

treatment of the extract with DNAse I (Takara). Quantitative real-time reverse transcriptase-PCR analysis was performed according to a previous study (12). Briefly, $500 \mathrm{ng}$ of total RNA was reverse transcribed to cDNA with Takara PCR Thermal Cycler Dice and then the cDNA was subjected to real-time PCR using the Applied Biosystems 7500 PCR Device (ABI7500, USA). Expression of Bax, caspase-3, cytochrome $\mathrm{c}$, and fas mRNA was normalized to GAPDH mRNA and the data were analyzed according to relative gene expression by the $2^{-\Delta \Delta C T}$ (Livak) method. The experiments were performed in triplicate and repeated once.

Statistical analysis. The data were summarized from at least three independent experiments. The results were expressed as the mean \pm standard deviation and evaluated using oneor two-way analysis of variance (ANOVA) followed by the Student's t-test. Statistical significance was defined as $\mathrm{P}<0.05$

\section{Results}

Allicin-induced reduction of SGC-7901 cancer cell viability. Viability of allicin-treated SGC-7901 cells was first assessed using the MTT assay. We found that allicin decreased tumor cell viability in a dose- and time-dependent manner (Fig. 1). Allicin induced the viable cells by $70.1 \pm 4.5 \%$ after 48 -h treatment with $30 \mu \mathrm{g} / \mathrm{ml}$ allicin (Fig. 1).

Allicin treatment induced apoptosis. We then determined the cause of the reduced cell viability by allicin treatment in gastric cancer cells. We first performed transmission electron microscope detection of morphology changes following treatment of the SGC-7901 cells with $30 \mu \mathrm{g} / \mathrm{ml}$ allicin for $48 \mathrm{~h}$ and found that the allicin-treated cancer cells had reduced size, increased chromatin condensation, cytoplasm density, cytoplasm vacillation, and marinated nuclear chromatin; cell nucleus was broken (Fig. 2B), suggesting that the cells under- 


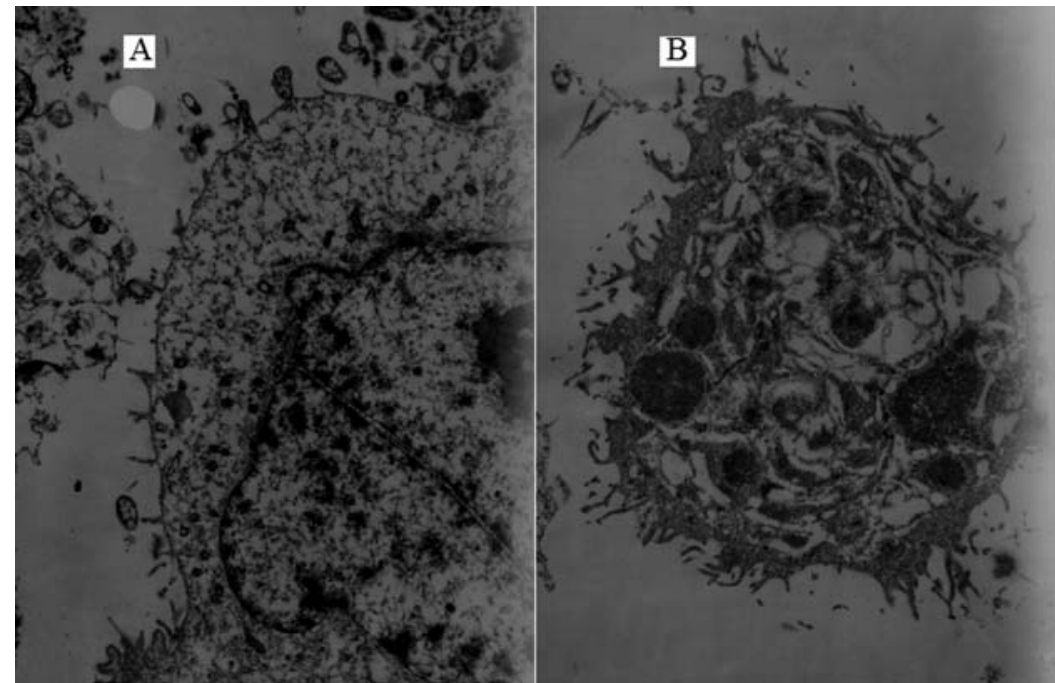

Figure 2. Transmission electron photomicrographs. The cells were grown and treated with $30 \mu \mathrm{g} / \mathrm{ml}$ allicin for $48 \mathrm{~h}$ and subjected to transmission electron microscope analysis of the altered morphology. (A) A control cell. (B) Allicin-treated cell.

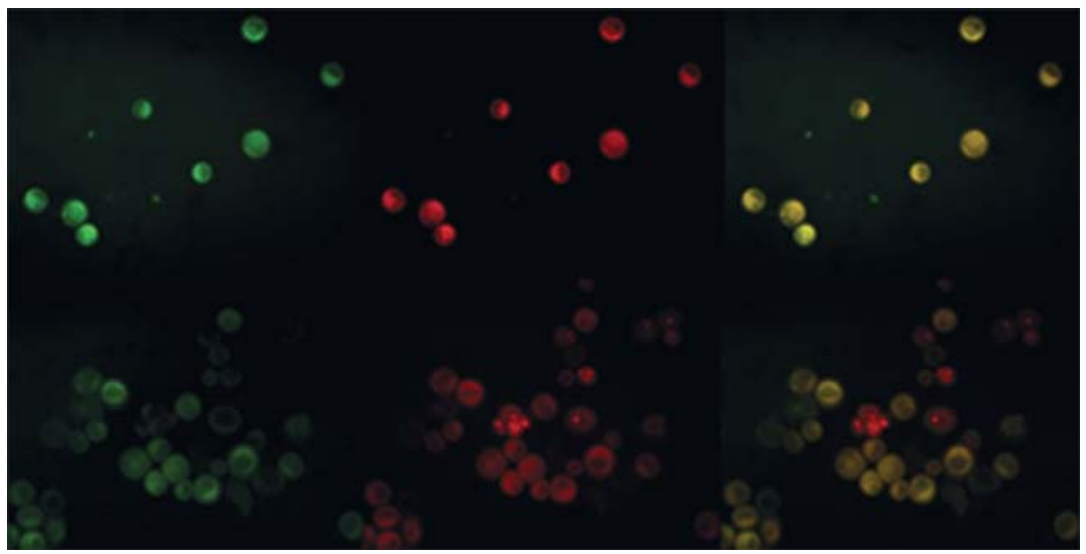

Figure 3. Rh123 and propidium iodide staining. The cells were grown and treated with or without $30 \mu \mathrm{g} / \mathrm{ml}$ allicin for $48 \mathrm{~h}$ and then stained with Rh123 and propidium iodide, followed by quantification using a fluorescence microscope.

went apoptosis. However, control cells showed normal nuclei and cytoplasm and microvillies on the cell surface (Fig. 2A).

Rh123 and propidium iodide staining of apoptotic cells. We chemically stained the gastric cancer cells with Rh123 and propidium iodide and found that after treatment with $30 \mu \mathrm{g} / \mathrm{ml}$ allicin for $48 \mathrm{~h}$, SGC-7901 cells showed chromatin condensation and fragmentation in the nuclei, and enhanced fluorescence as distinctive morphological characteristic of apoptotic cells, whereas the control cells were not different (Fig. 3).

Annexin V/FITC detection of apoptosis. We then performed an Annexin V/FITC assay followed by flow cytometry to distinguish apoptosis and necrosis following allicin treatment. Our data showed that the production of apoptotic cells was increased to $15.14 \pm 1.5 \%$ after treatment with $30 \mu \mathrm{g} / \mathrm{ml}$ allicin for $48 \mathrm{~h}$ compared with control cells $2.48 \pm 0.5 \%$, indicating that allicin might also induce cells to undergo necrosis in addition to induction of apoptosis (Fig. 4).

Changes in the mitochondrial membrane potential by allicin. Activation of mitochondrial signaling pathways is involved in the change of the outer mitochondrial membrane permeabilization by subsequently releasing pro-apoptotic factors, such as cytochrome c, into the cytosol. We determined changes in mitochondrial membrane potential following allicin treatment and demonstrate in Fig. 5 that control cells showed strong staining for Rh123, indicating a negative membrane potential of the mitochondria and the accumulation of the lipophilic dye, whereas allicin-treated cells were depolarized and the cell population was shifted to the left, indicating that allicin induced significant disruption of the mitochondrial potential membrane potential $(\Delta \Psi \mathrm{m})$.

Release of cytochrome $c$ into the cytoplasm and activation of caspase-3 by allicin treatment. Our data so far, demonstrated that allicin induced gastric cancer cells to undergo apoptosis. Next, we determined the molecular mechanisms responsible for the effects of allicin in tumor cells. We first analyzed the release of cytochrome $\mathrm{c}$ from the mitochondria and then the activation of caspase- 3 in the cells following treatment with or without allicin. We found that allicin treatment induced cytochrome c release form the mitochondria, which was detected by immunocytochemistry (Fig. 6A), Western blot 
A

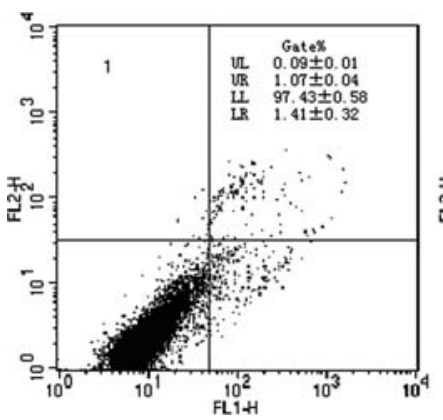

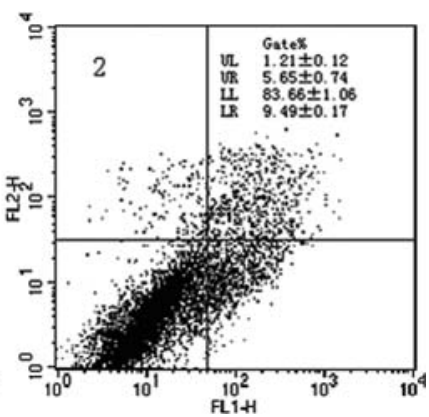

B

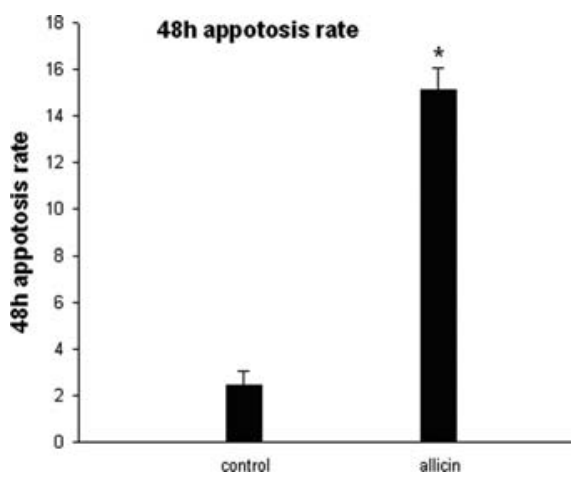

Figure 4. Annexin V/FITC assay. The cells were grown and treated with or without $30 \mu \mathrm{g} / \mathrm{ml}$ allicin for $48 \mathrm{~h}$ and then subjected to Annexin V/FITC staining and flow cytometry. (A) The horizontal (FL1-H) and vertical (FL2-H) axes represent positive labeling of Annexin V/FITC/PI. (B) Quantified data from A. The data represent means \pm SEM $(n=6)$ of three experiments. ${ }^{*} \mathrm{P}<0.05$ as compared with control.

A
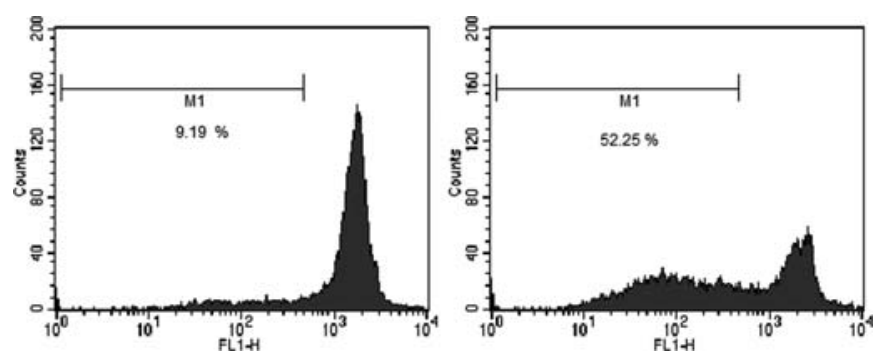

B

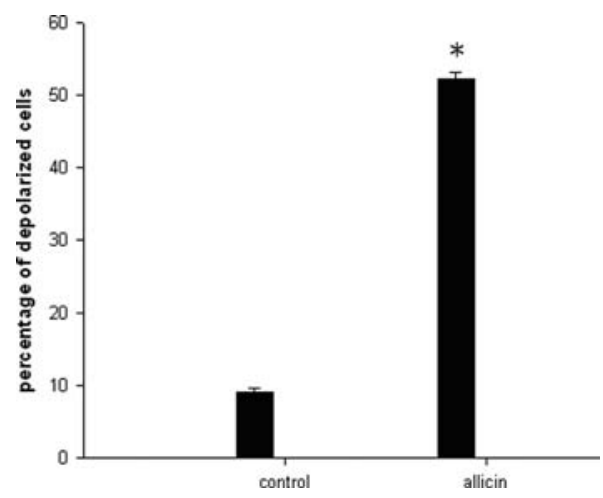

Figure 5. Allicin-induced disruption of the mitochondrial membrane potential $(\Delta \Psi \mathrm{m})$. (A) SGC-7901 cells were treated with allicin (30 $\mu \mathrm{g} / \mathrm{ml})$ for $48 \mathrm{~h}$ and then stained with rhodamine 123 for flow cytometric analysis. Left, control cells; right, allicin-treated cells. (B) Quantitative data from A. *P<0.05 vs. the control group. All experiments were performed more than three times with similar results.

A

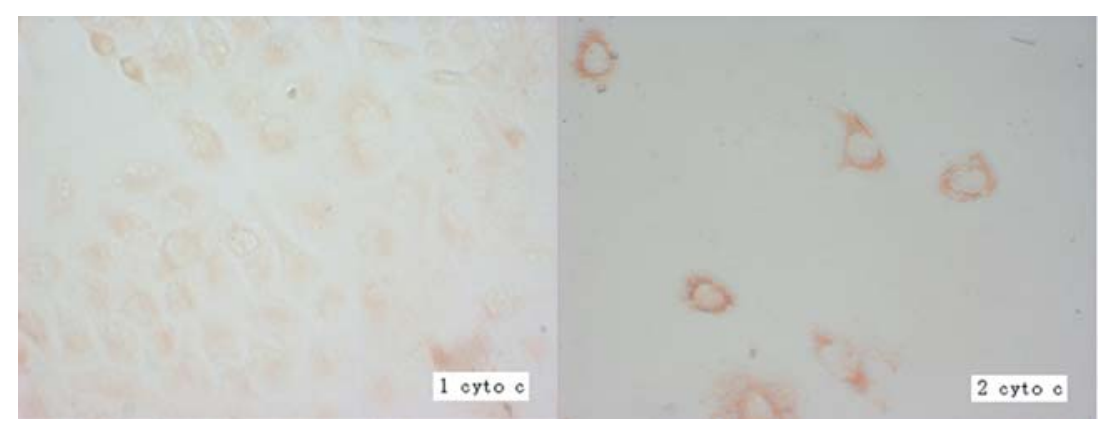

B

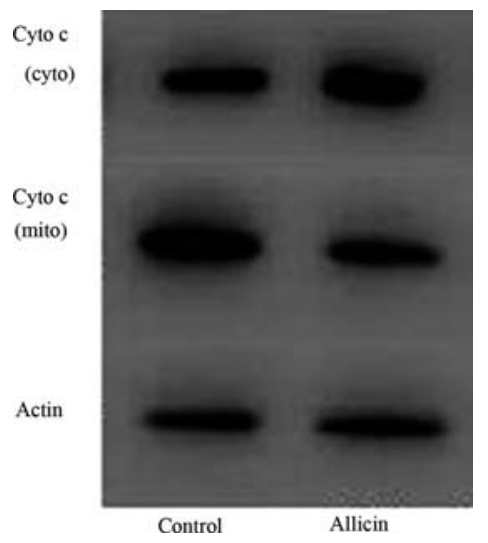

C

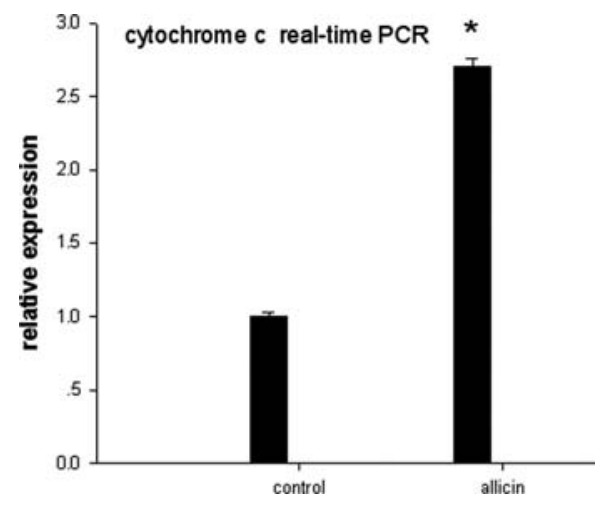

Figure 6. Allicin-induced cytochrome c release from the mitochondria. The cells were grown and treated with or without $30 \mu \mathrm{g} / \mathrm{m} 1$ allicin for $48 \mathrm{~h}$ and then subjected to immunocytochemical (A), Western blot analysis (B), and Q-RT-PCR analyses (C). 
A

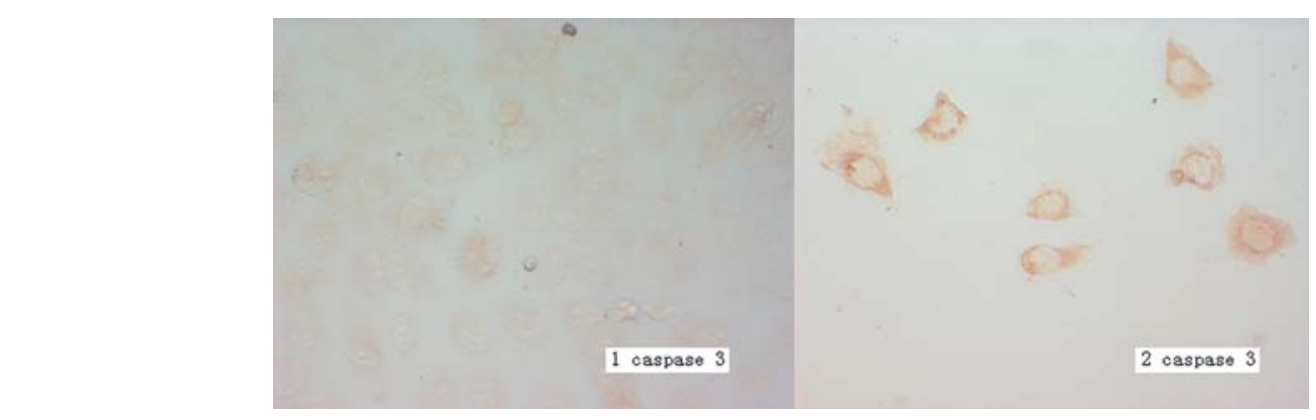

B

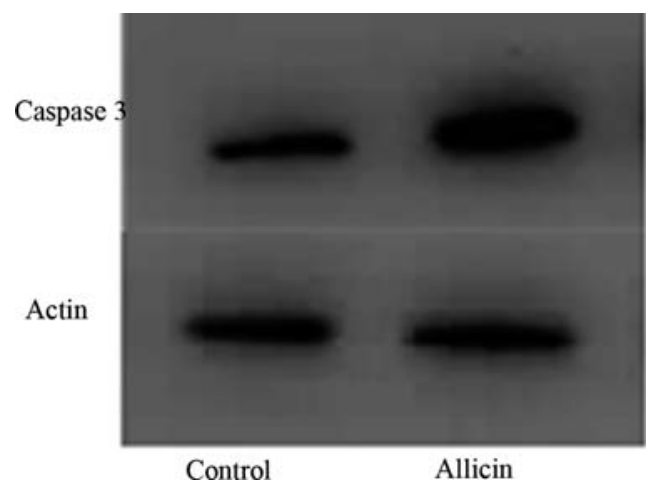

C

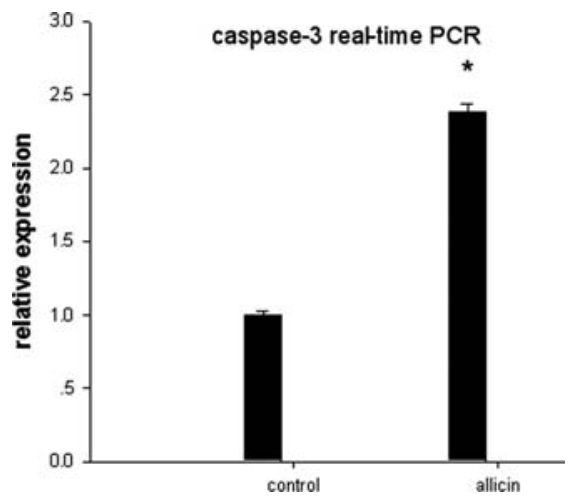

Figure 7. Allicin-induced caspase-3 activation. The cells were grown and treated with or without $30 \mu \mathrm{g} / \mathrm{m} 1$ allicin for $48 \mathrm{~h}$ and then subjected to immunocytochemical (A), Western blot analysis (B), and Q-RT-PCR analyses (C).

A

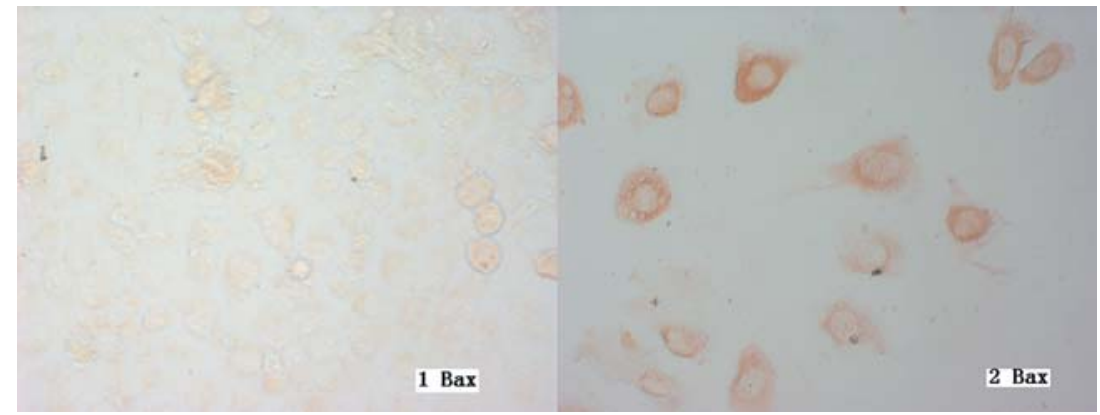

B

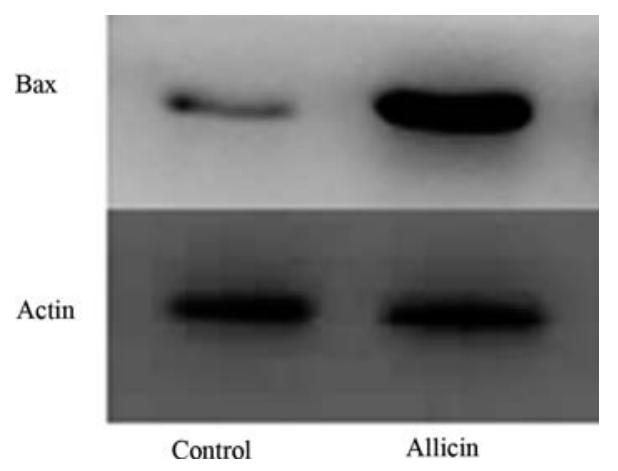

C

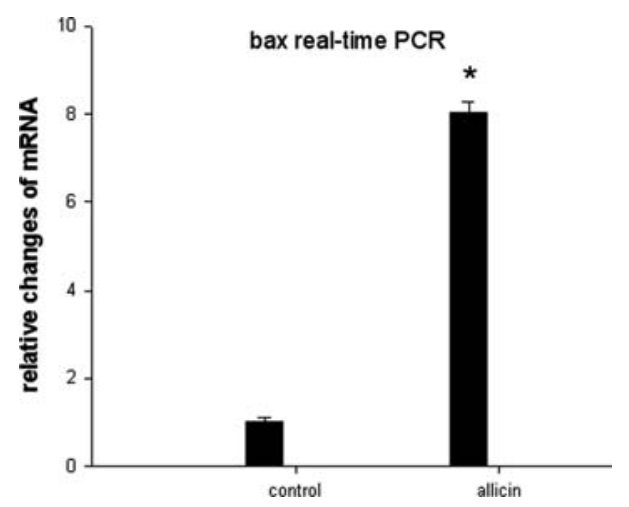

Figure 8. Allicin induction of bax expression. The cells were grown and treated with or without $30 \mu \mathrm{g} / \mathrm{m} 1$ allicin for $48 \mathrm{~h}$ and then subjected to immunocytochemical (A), Western blot analysis (B), and Q-RT-PCR analyses (C).

analysis (Fig. 6B), and Q-RT-PCR (Fig. 6C). In addition, caspase- 3 was also activated in the allicin-treated SGC-7901 cells detected by immunocytochemistry (Fig. 7A), Western blot analysis (Fig. 7B), and Q-RT-PCR (Fig. 7C).

Allicin induction of Bax expression in SGC-7901 cells. We then investigated more mitochondrial apoptosis-related genes, such as bax following allicin treatment. We found that allicin induced expression of bax proteins and mRNA detected by immunocytochemical (Fig. 8A), Western blot analysis (Fig. 8B), and Q-RT-PCR analyses (Fig. 8C).

Allicin induction of Fas expression in SGC-7901 cells. Fas expression was induced by allicin treatment in SGC-7901 
A

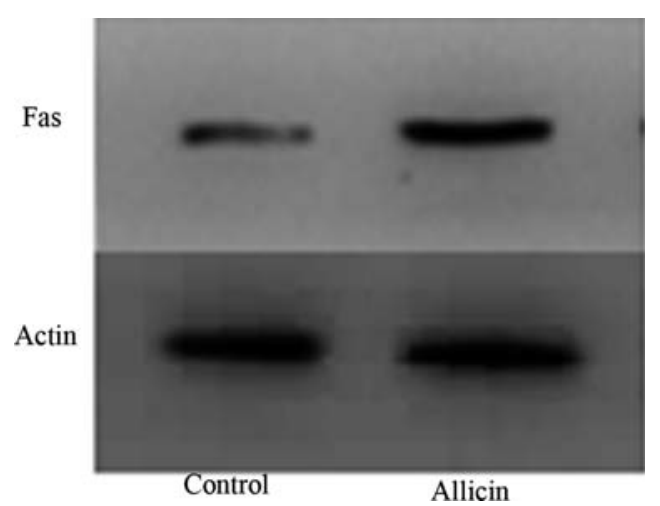

B

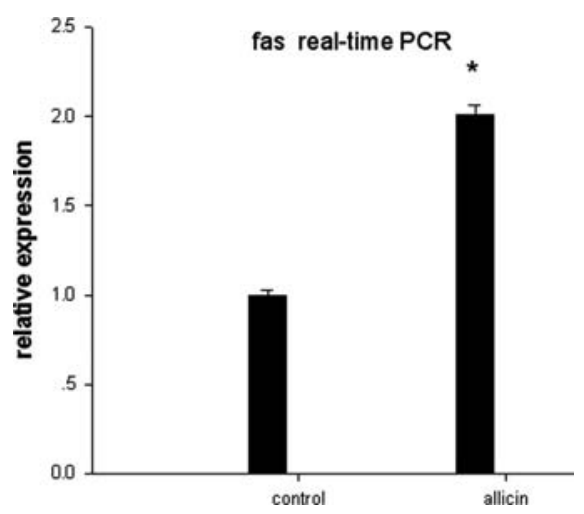

Figure 9. Allicin induction of fas expression. The cells were grown and treated with or without $30 \mu \mathrm{g} / \mathrm{m} 1$ allicin for $48 \mathrm{~h}$ and then subjected to Western blot analysis (A) and Q-RT-PCR analyses (B).

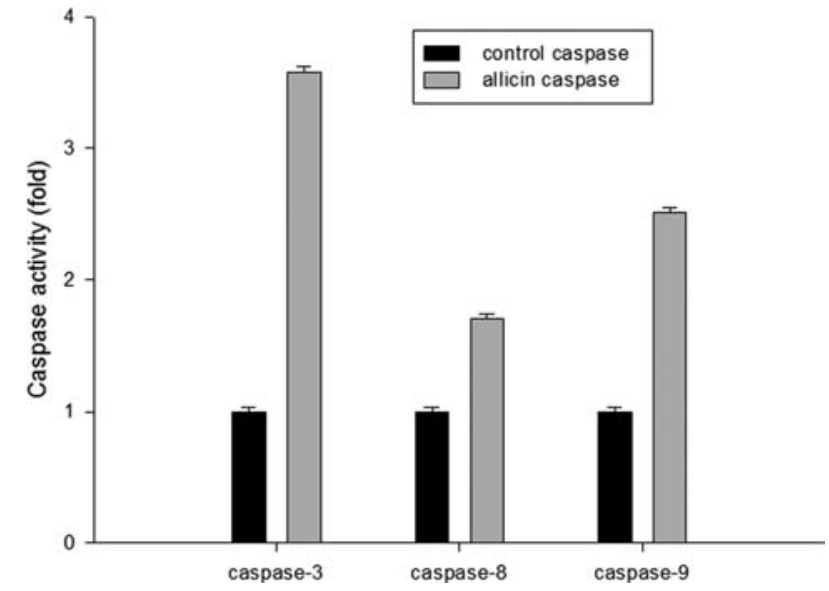

Figure 10. Allicin induction of caspase-3, -8, and -9 activation. The cells were grown and treated with or without $30 \mu \mathrm{g} / \mathrm{m} 1$ allicin for $48 \mathrm{~h}$ and then subjected to flow cytometry analysis. ${ }^{*} \mathrm{P}<0.05$ vs. the control cells.

cells detected by Western blot analysis (Fig. 9A) and Q-RTPCR analyses (Fig. 9B).

Allicin activation of caspase enzymes. We detected caspase-3, -8 , and -9 activation following allicin treatment in gastric cancer cells and found that the activity of caspase-3, -8 , and -9 were all significantly increased following allicin treatment for $48 \mathrm{~h}$ (Fig. 10).

\section{Discussion}

In this study, we detected the effects of allicin on gastric cancer cells and determined the underlying molecular mechanisms involved. We found that allicin treatment reduced cell viability due to the induction of apoptosis in tumor cells, which was evident using transmission electron microscopy, Rh123 and propidium iodide staining, the Annexin V/FITC assay, and changes in the mitochondrial membrane potential. Molecularly, allicin induced cytochrome c release from the mitochondria and increased caspase- $3,-8$, and -9 activation through via induction of bax and fas expression. This study indicates the usefulness of allicin in the control of gastric cancer and further investigations will likely extend these findings to other cancer types.
To date, prevention and effective chemotherapy for gastric cancer has become important for patients suffering from cancer, particularly since this cancer is usually found at the later stages at diagnosis and lacks effective drugs for therapy $(1,2)$. The data from our current study showed the usefulness and promise of allicin treatment to reduce gastric cancer cell viability and undergo apoptosis. In particular, $30 \mu \mathrm{g} / \mathrm{ml}$ allicin treatment for $48 \mathrm{~h}$ reduced tumor cell viability by $70.1 \pm 4.5 \%$ and therefore, allicin should be further developed as a novel agent in the control of gastric cancer.

Apoptosis involves a series of biochemical events leading to a characteristic cell morphology and cell death. Two main molecular signaling pathways have been characterized during cell apoptosis (13-17). The extrinsic pathway is initiated through ligand stimulation of the cell surface death receptors, such as TNFR or CD95R. CD95R is a transmembrane protein with an extracellular domain rich in cysteins that bind the Fas ligand (FasL), and the cytoplasm domain involved in death signaling (15). After the binding of Fas to FasL, trimerization and recruitment of Fas-associated death domain (FADD) proteins through homotypic death domain interactions occurs. In turn, trimerized FADD recruits either procaspase- 8 or -10 leading to autoproteolysis and causing activation (16). Caspase- 8 interacts with procaspase-3, -6 , or -7 and, through a process of trans-proteolysis, activates the latter caspases for cleavage of genomic DNA in the cells.

However, the intrinsic pathway is triggered by mitochondrial stress caused by various factors, such as DNA damage, oxidative stress and heat shock proteins (reviewed in ref. 17). This pathway is initiated through the release of signaling factors from the mitochondria as a consequence of mitochondrial membrane permeability transition. Such changes lead to translocation of pro- and antiapoptotic proteins across the mitochondrial membranes $(18,19)$. Cytochrome $\mathrm{c}$ is the first to be released from the mitochondria into the cytosol and binds to Apaf-1 and ATP, which then binds to procaspase- 9 to create a protein complex, which activates caspase-9, and then eventually activates caspase- 3 for visualizing apoptotic phenotypes. In addition, Bax and $\mathrm{Bcl}-2$ proteins will affect cytochrome c release.

Several other studies have been conducted to assess the effects of allicin and the underlying molecular mechanisms. For example, in vitro studies of allicin treatment on human 
mammary MCF-7 cancer cells revealed that its antiproliferative activity is accompanied by accumulation of cells in the regulatory checkpoints, G0/G1 and G2/M, of the cell cycle $(10,20)$. Allicin was shown to induce apoptosis in gastric cancer cells, partly by decreased telomerase activity (21). Allicin was also reported to induce apoptosis in human epithelial carcinoma through a caspase-independent pathway, mediated by the release of apoptotic-inducing factor (AIF) from the mitochondria and protein kinase A (PKA) activation (22), or potentially through the mitochondrial pathway (23-25).

In summary, we have demonstrated that allicin treatment inhibited proliferation and induced apoptosis in SGC-7901 cancer cells. Both intrinsic mitochondrial and extrinsic Fas/ FasL-mediated pathways of apoptosis occur simultaneously in SGC-7901 cells following allicin treatment. This finding suggests that allicin is a multi-targeted or multi-functional compound in the control of gastric cancer cells and suggests that allicin may have the potential to inhibit tumor growth in vivo. Future studies should test the cytotoxicity of allicin in normal cells and further evaluate it as a novel antitumor agent for the control of gastric cancer.

\section{Acknowledgements}

This study is supported by the Foundation of the Excellent Youth in Liaoning Province (No. 2006R54) and in part by a grant from the Science and Technology Program, Liaoning Province of China (No. 2007225005-13).

\section{References}

1. Hohenberger P and Gretschel S: Gastric cancer. Lancet 362: 305-315, 2003

2. Leung WK, Wu MS, Kakugawa Y, et al: Screening for gastric cancer in Asia: current evidence and practice. Lancet Oncol 9: 279-287, 2008

3. Herman-Antosiewicz A and Singh SV: Signal transduction pathways leading to cell cycle arrest and apoptosis induction in cancer cells by Allium vegetable-derived organosulfur compounds: a review. Mutat Res 555: 121-131, 2004.

4. Xiao D, Lew KL, Kim YA, et al: Diallyl trisulfide suppresses growth of PC-3 human prostate cancer xenograft in vivo in association with Bax and Bak induction. Clin Cancer Res 12: 6836-6843, 2006

5. Xiao XL, Peng J, Su Q, et al: Diallyl trisulfide induces apoptosis of human gastric cancer cell line MGC803 through caspase-3 pathway. Ai Zheng 25: 1247-1251, 2006.

6. Antosiewicz J, Herman-Antosiewicz A, Marynowski SW and Singh SV: c-Jun NH(2)-terminal kinase signaling axis regulates diallyl trisulfide-induced generation of reactive oxygen species and cell cycle arrest in human prostate cancer cells. Cancer Res 66: , 2006.
7. Jakubikova J and Sedlak J: Garlic-derived organosulfides induce cytotoxicity, apoptosis, cell cycle arrest and oxidative stress in human colon carcinoma cell lines. Neoplasma 53: 191-199, 2006.

8. Fujisawa H, Watanabe K, Suma K, et al: Antibacterial potential of garlic-derived allicin and its cancellation by sulfhydryl compounds. Biosci Biotechnol Biochem 73: 1948-1955, 2009.

9. Peter KV: Handbook of herbs and spices. CRC Press LLC, Boca Raton, FL, pp193-207, 2000

10. Chen TJ, Ha MW, Gong YH, Qian X and Yuan Y: Effect of allitridi on inducing mitotic arrest in human gastric cell line SGC-7901 and its possible mechanism. Chin J Cancer Res 20: 126-132, 2008

11. Chen Z, Merta PJ, Lin NH, et al: A-432411, a novel indolinone compound that disrupts spindle pole formation and inhibits human cancer cell growth. Mol Cancer Ther 4: 562-568, 2005.

12. Chomczynski P: A reagent for the single-step simultaneous isolation of RNA, DNA and proteins from cell and tissue samples. Biotechniques 15: 532-537, 1993.

13. Roy MK, Thalang VN, Trakoontivakorn G and Nakahara K: Mahanine, a carbazole alkaloid from Micromelum minutum, inhibits cell growth and induces apoptosis in U937 cells through a mitochondrial dependent pathway. Br J Pharmacol 145: 145-155, 2005.

14. Cory S and Adams JM: The Bcl2 family: regulators of the cellular life-or-death switch. Nat Rev Cancer 2: 647-656, 2002.

15. Henkler F, Behrle E, Dennehy KM, et al: The extracellular domains of FasL and Fas are sufficient for the formation of supramolecular FasL-Fas clusters of high stability. J Cell Biol 168: 1087-1098, 2005.

16. Carrington PE, Sandu C, Wei Y, et al: The structure of FADD and its mode of interaction with procaspase-8. Mol Cell 22: 599-610, 2006.

17. Jin Z and El-Deiry WS: Overview of cell death signaling pathways. Cancer Biol Ther 4: 139-163, 2005.

18. Hsu YT, Wolter KG and Youle RJ: Cytosol-to-membrane redistribution of $\mathrm{Bax}$ and $\mathrm{Bcl}-\mathrm{X}(\mathrm{L})$ during apoptosis. Proc Natl Acad Sci USA 94: 3668-3672, 1997.

19. Wolter KG, Hsu YT, Smith CL, Nechushtan A, Xi XG and Youle RJ: Movement of Bax from the cytosol to mitochondria during apoptosis. J Cell Biol 139: 1281-1292, 1997.

20. Hirsch K, Danilenko M, Giat J, et al: Effect of purified allicin, the major ingredient of freshly crushed garlic, on cancer cell proliferation. Nutr Cancer 38: 245-254, 2000.

21. Sun L and Wang X: Effects of allicin on both telomerase activity and apoptosis in gastric cancer SGC-7901 cells. World J Gastroenterol 9: 1930-1934, 2003.

22. Park SY, Cho SJ, Kwon HC, Lee KR, Rhee DK and Pyo S: Caspase-independent cell death by allicin in human epithelial carcinoma cells: involvement of PKA. Cancer Lett 224: 123-132, 2005.

23. Miron T, Wilchek M, Sharp A, et al: Allicin inhibits cell growth and induces apoptosis through the mitochondrial pathway in HL60 and U937 cells. J Nutr Biochem 19: 524-535, 2008.

24. Nagaraj NS, Anilakumar KR and Singh OV: Diallyl disulfide causes caspase-dependent apoptosis in human cancer cells through a Bax-triggered mitochondrial pathway. J Nutr Biochem 21: 405-412, 2010.

25. Yang JS, Chen GW, Hsia TC, et al: Diallyl disulfide induces apoptosis in human colon cancer cell line (COLO 205) through the induction of reactive oxygen species, endoplasmic reticulum stress, caspases casade and mitochondrial-dependent pathways. Food Chem Toxicol 47: 171-179, 2009. 